UCRL-JC-127986

PREPRINT

\title{
Kinetic Calculations of Explosives with Slow-Burning Constituents
}

\author{
W. Michael Howard \\ P. Clark Souers \\ Laurence E. Fried
}

This paper is prepared for submittal to the

1997 Topical Conference on Shock Compression of Condensed Matter July 27-Aug. 1, 1997, Univ. of Massachusetts, Amherst, MA.

July 1997

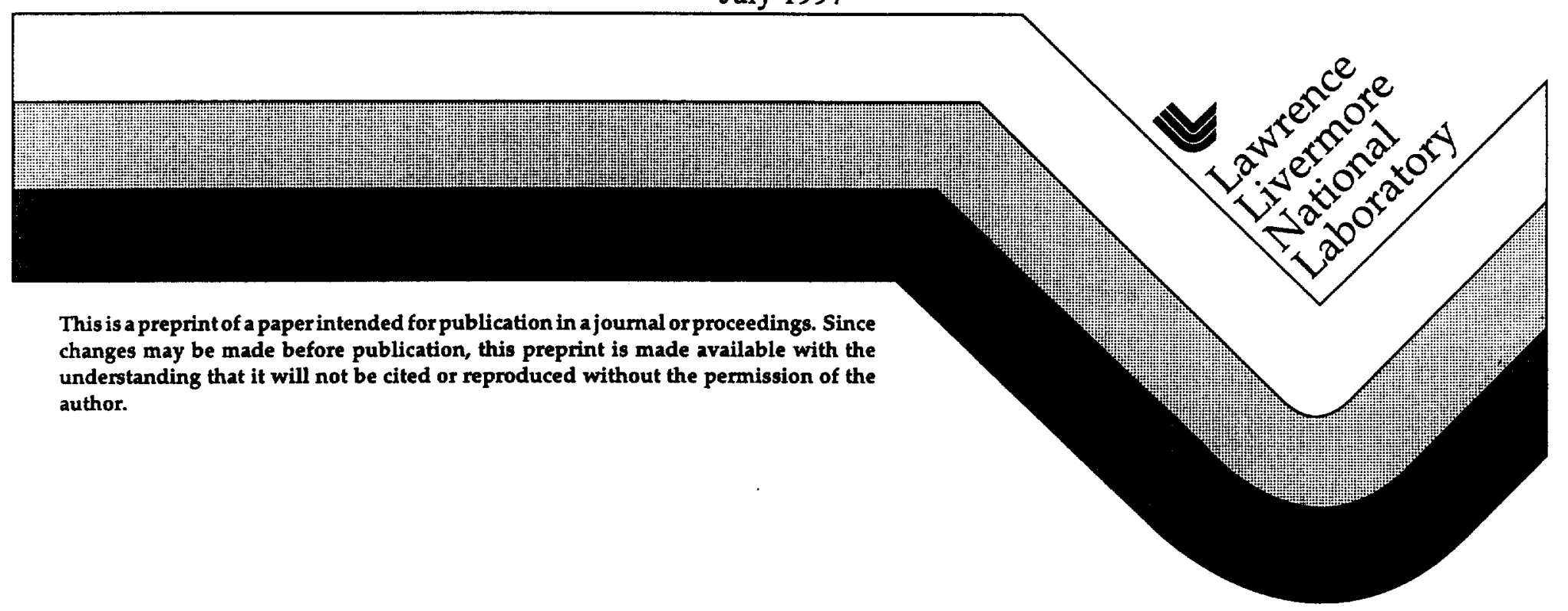




\section{DISCLAIMER}

This document was prepared as an account of work sponsored by an agency of the United States Government. Neither the United States Government nor the University of California nor any of their employees, makes any warranty, express or implied, or assumes any legal liability or responsibility for the accuracy, completeness, or usefulness of any information, apparatus, product, or process

disclosed, or represents that its use would not infringe privately owned rights. Reference herein to any specific commercial product, process, or service by trade name, trademark, manufacturer, or otherwise, does not necessarily constitute or imply its endorsement, recommendation, or favoring by the United States Government or the University of California. The views and opinions of authors expressed herein do not necessarily state or reflect those of the United States Government or the University of California, and shall not be used for advertising or product endorsement purposes. 


\title{
KINETIC CALCULATIONS OF EXPLOSIVES WITH SLOW-BURNING CONSTITUENTS
}

\author{
W. Michael Howard, P. Clark Souers and Laurence E. Fried \\ Energetic Materials Center, Lawrence Livermore National Laboratory \\ Livermore, CA 94550 USA
}

The equilibrium thermochemical code CHEETAH V1.40 has been modified to detonate part of the explosive and binder. An Einstein thermal description of the unreacted constituents is used, and the Einstein temperature may be increased to reduce heat absorption. We study the effect of the reactivity and thermal transport on the detonation velocity. Hydroxy-terminated-polybutadiene binders have low energy and density and would degrade the detonation velocity if they burned. Runs with unburned binder are closer to the measured values. Aluminum and ammonium perchlorate are also largely unburned within the sonic reaction zone that determines the detonation velocity. All three materials appear not to fully absorb heat as well. The normal assumption of total reaction in a thermochemical code is clearly not true for these special cases, where the detonation velocities have widely different values for different combinations of processes.

The detonation velocity of an explosive is usually calculated in a thermochemical code with the assumption of full chemical and thermal equilibrium. This implies that all products are consumed in the detonation wave. This assumption holds in the limit of an infinite-size sample, whereas actual finite cylinders may give different detonation velocities. This arises because of the size effect: some components of the explosive react too late to drive the detonation front. Also, heat may flow too slowly to bring all components into thermal equilibrium.

It is possible to study the results of the size effect with an equilibrium thermochemical code as long as the various possibilities give widely different predictions for the detonation velocity. Here, we use the equilibrium thermochemical code CHEETAH plus the V1.40 BKWC library $[1,2]$, which can make some or all of the starting materials inert. If a particular component reacts, it is converted into gas at the temperature of the overall explosive, whether it has chemical energy to give or not. If it does not react, it takes up volume according to a specified equation-of-state. For the heat capacity we use the Einstein model. By setting the Einstein temperature to $10^{5} \mathrm{~K}$, the material has little heat capacity at detonation temperatures $(3000-5000 \mathrm{~K})$. In effect, we have decoupled it thermally from the hot product gases. Thus, we can have a component that does not react and also absorbs no heat from the product gases.

Also included in this version of CHEETAH is the equation-of-state of the unreacted explosive, where the temperature-independent part of the equation of state is represented by a Murnaghan form [4]. The key is to find initial components such that the degree of reaction and/or thermal transport changes the detonation velocity substantially. The first three samples in Table 1 include large quantities 
of water which does not react [5]. The results suggest that the water does not transmit the heat of the detonating explosive either.

Finding an illustrative binder is more difficult, but Lawrence has been treating hydroxyterminated-butadiene (HTPB) as inert in thermochemical codes for some time [6]. It has a low density of $0.907 \mathrm{~g} / \mathrm{cc}, \mathrm{a}-0.159 \mathrm{~kJ} / \mathrm{mol}$ heat of formation and the composition $\mathrm{C}_{7.33} \mathrm{H}_{11} \mathrm{O}_{0.083}$ [7]. Listed densities vary by less than $1 \%$ and the heat of formation of HTPB polymers used in explosives varies from -12 to $+63 \mathrm{~kJ} / 100$ grams [8]. In the explosive, HTPB is cured to a rubber and there is every expectation of uniform distribution. Its unreacted EOS is [9]

$$
\mathrm{U}_{\mathrm{s}}(\mathrm{mm} / \mu \mathrm{s})=1.63+2.24 \mathrm{u}_{\mathrm{p} .}(1)
$$

Six HTPB explosives are listed in Table 1 with combinations of reactivity and thermal transport on and off [10-14]. The results show best agreement with no reactivity of the binder as it affects the detonation velocity, and the spread of detonation velocities is large enough, i.e. greater than about $\pm 0.2 \mathrm{~mm} / \mu \mathrm{s}$, that we feel confident that the binder is not consumed ahead of the sonic point. The results also suggest that heat transfer may take place in the HTPB. The final samples in Table 1 are aluminum and ammonium perchlorate.[15-16] The aluminum shows no reactivity or heat transfer. The AP shows no reactivity but perhaps some heat transfer.

For effective heat transfer, the heat diffusivity timescale must be much shorter than the sonic reaction zone timescale. The heat diffusivity depends on the thermal conductivity, the particle size, the density and the heat capacity. We estimate that to obtain heat transfer with HTPB binder, we need $0.2 \mu \mathrm{m}$ particle size, while for AP we require $1.0 \mu \mathrm{m}$ particle size or less. Aluminum has the highest diffusivity, but the particles are typically large, so that heat transfer does not occur.

Finally, we consider the heat effects of the shock wave as estimated from the Hugoniots. A shock wave of $30 \mathrm{GPa}$ from the explosive will cause compressive heating of: $550 \mathrm{~K}$ in $\mathrm{Al}, 1500 \mathrm{~K}$ in kel$\mathrm{F}$, and $2500 \mathrm{~K}$ in AP. Given explosive temperatures of $3000-4000 \mathrm{~K}$, This suggests that shock heating may transfer some of the heat in AP and kel-F but almost none in aluminum.
This work was performed under the auspices of the US Department of Energy by the Lawrence Livermore National Laboratory under contract number W-7405ENG-48 
TABLE 1. Calculated and measured detonation velocities for various explosives where some secondary component does not burn within the reaction zone.

\begin{tabular}{|c|c|c|c|c|c|c|}
\hline \multirow[b]{2}{*}{$\begin{array}{l}\text { Type of } \\
\text { Sample }\end{array}$} & \multirow{2}{*}{$\begin{array}{l}\text { Explosive } \\
\text { Binder } \\
\text { Density }\end{array}$} & \multicolumn{2}{|c|}{ Secondary Material } & \multirow{2}{*}{$\begin{array}{c}\text { Det } \\
\text { Velocity } \\
(\mathrm{mm} / \mu \mathrm{s}) \\
\end{array}$} & \multirow{2}{*}{$\begin{array}{l}\text { Composi- } \\
\text { tion } \\
\text { (wt \%) }\end{array}$} & \multirow{2}{*}{$\begin{array}{l}\text { Conclusion } \\
\text { Reactivity } \\
\text { Thermal }\end{array}$} \\
\hline & & Reactivity & $\begin{array}{l}\text { Thermal } \\
\text { Transport }\end{array}$ & & & \\
\hline $\begin{array}{l}\text { No } \\
\text { chemical } \\
\text { reaction }\end{array}$ & $\begin{array}{l}\mathrm{HMX} \\
\text { water } \\
1.43 \mathrm{~g} / \mathrm{cc} \\
\end{array}$ & $\begin{array}{l}\text { off } \\
\text { Measured } \\
\text { off }\end{array}$ & $\begin{array}{l}\text { on } \\
\text { off } \\
\end{array}$ & $\begin{array}{l}6.47 \\
7.06 \\
7.42 \\
\end{array}$ & $\begin{array}{c}\text { HMX } 64 \\
\text { water } 36\end{array}$ & $\begin{array}{l}\text { thermal } \\
\text { partly } \\
\text { on }\end{array}$ \\
\hline & $\begin{array}{l}\text { HMX } \\
\text { water } \\
1.54-1.55 \mathrm{~g} / \mathrm{cc}\end{array}$ & $\begin{array}{l}\text { off } \\
\text { Measured } \\
\text { off }\end{array}$ & $\begin{array}{l}\text { on } \\
\text { off }\end{array}$ & $\begin{array}{l}7.45 \\
7.96 \\
7.99\end{array}$ & $\begin{array}{l}\text { HMX } 80 \\
\text { water } 20\end{array}$ & $\begin{array}{l}\text { thermal } \\
\text { off }\end{array}$ \\
\hline & $\begin{array}{l}\mathrm{RX}-23-\mathrm{AB} \\
\text { water } \\
1.356 \mathrm{~g} / \mathrm{cc} \\
\end{array}$ & $\begin{array}{l}\text { off } \\
\text { off } \\
\text { Measured }\end{array}$ & $\begin{array}{l}\text { on } \\
\text { off }\end{array}$ & $\begin{array}{l}6.51 \\
7.06 \\
7.48 \\
\end{array}$ & $\begin{array}{l}\text { hyd nit } 69 \\
\text { hyd } 5 \\
\text { water } 26 \\
\end{array}$ & $\begin{array}{l}\text { thermal } \\
\text { off }\end{array}$ \\
\hline \multirow[t]{5}{*}{$\begin{array}{l}\text { HTPB } \\
\text { binders }\end{array}$} & $\begin{array}{l}\mathrm{B} 2141 \\
1.63 \mathrm{~g} / \mathrm{cc}\end{array}$ & $\begin{array}{l}\text { on } \\
\text { off } \\
\text { Measured } \\
\text { off } \\
\end{array}$ & $\begin{array}{l}\text { on } \\
\text { on } \\
\text { off } \\
\end{array}$ & $\begin{array}{l}7.56 \\
8.16 \\
8.19 \\
8.80 \\
\end{array}$ & $\begin{array}{l}\text { RDX 88 } \\
\text { HTPB } 12\end{array}$ & off/on \\
\hline & $\begin{array}{l}\mathrm{P} 2100 \mathrm{~B} \\
1.70 \mathrm{~g} / \mathrm{cc}\end{array}$ & $\begin{array}{l}\text { on } \\
\text { off } \\
\text { Measured } \\
\text { off }\end{array}$ & $\begin{array}{l}\text { on } \\
\text { on } \\
\text { off }\end{array}$ & $\begin{array}{l}7.80 \\
8.51 \\
8.57 \\
9.12 \\
\end{array}$ & $\begin{array}{l}\text { HMX } 88 \\
\text { HTPB } 12\end{array}$ & off/on \\
\hline & $\begin{array}{l}\mathrm{A}-589 \\
1.66 \mathrm{~g} / \mathrm{cc}\end{array}$ & $\begin{array}{l}\text { on } \\
\text { Measured } \\
\text { off } \\
\text { off }\end{array}$ & $\begin{array}{l}\text { on } \\
\text { on } \\
\text { off }\end{array}$ & $\begin{array}{l}7.55 \\
8.26 \\
8.31 \\
9.04 \\
\end{array}$ & $\begin{array}{l}\text { HMX } 86 \\
\text { HTPB } 14\end{array}$ & off/on \\
\hline & $\begin{array}{l}\mathrm{HX}-72 \\
1.48 \mathrm{~g} / \mathrm{cc}\end{array}$ & $\begin{array}{l}\text { on } \\
\text { off } \\
\text { Measured } \\
\text { off }\end{array}$ & $\begin{array}{l}\text { on } \\
\text { on } \\
\text { off }\end{array}$ & $\begin{array}{l}6.65 \\
7.31 \\
7.75 \\
8.41 \\
\end{array}$ & $\begin{array}{l}\text { RDX 80 } \\
\text { HTPB 20 }\end{array}$ & $\begin{array}{l}\text { off } \\
\text { mostly } \\
\text { on }\end{array}$ \\
\hline & $\begin{array}{l}\text { IRX-1 } \\
1.43 \mathrm{~g} / \mathrm{cc}\end{array}$ & $\begin{array}{l}\text { on } \\
\text { off } \\
\text { Measured } \\
\text { off }\end{array}$ & $\begin{array}{l}\text { on } \\
\text { on } \\
\text { off }\end{array}$ & $\begin{array}{l}6.42 \\
6.95 \\
7.67 \\
8.50 \\
\end{array}$ & $\begin{array}{l}\text { HMX 70 } \\
\text { HTPB } 30\end{array}$ & $\begin{array}{l}\text { offf } \\
\text { partly } \\
\text { on }\end{array}$ \\
\hline $\begin{array}{l}\text { HTPB with } \\
\text { some } \mathrm{Al}\end{array}$ & $\begin{array}{l}\text { IRX-3A } \\
1.58 \mathrm{~g} / \mathrm{cc}\end{array}$ & $\begin{array}{l}\text { both on } \\
\text { both off } \\
\text { Measured } \\
\text { both off }\end{array}$ & $\begin{array}{l}\text { both on } \\
\text { both on } \\
\text { both off }\end{array}$ & $\begin{array}{l}7.08 \\
7.75 \\
7.87 \\
9.49 \\
\end{array}$ & $\begin{array}{l}\text { HMX } 58.5 \\
\text { HTPB } 35.6\end{array}$ & $\begin{array}{l}\text { offf } \\
\text { on }\end{array}$ \\
\hline $\begin{array}{l}\text { Al only; } \\
\text { no binders }\end{array}$ & $\begin{array}{l}\text { Tritonal } \\
1.695 \mathrm{~g} / \mathrm{cc}\end{array}$ & $\begin{array}{l}\text { on } \\
\text { off } \\
\text { off } \\
\text { Measured }\end{array}$ & $\begin{array}{l}\text { on } \\
\text { on } \\
\text { off }\end{array}$ & $\begin{array}{l}5.83 \\
6.20 \\
6.44 \\
6.52\end{array}$ & $\begin{array}{l}\text { TNT } 80 \\
\text { Al } 20\end{array}$ & offfoff \\
\hline & $\begin{array}{l}\text { TNM/Al } \\
1.828 \mathrm{~g} / \mathrm{cc}\end{array}$ & $\begin{array}{l}\text { off } \\
\text { on } \\
\text { Measured } \\
\text { off }\end{array}$ & $\begin{array}{l}\text { on } \\
\text { on } \\
\text { off }\end{array}$ & $\begin{array}{l}5.54 \\
5.73 \\
6.01 \\
6.02 \\
\end{array}$ & $\begin{array}{l}\text { TNM } 67.7 \\
\text { Al } 32.3\end{array}$ & offloff \\
\hline $\begin{array}{l}\text { high } \mathrm{Al} \text {; } \\
\text { other } \\
\text { materials }\end{array}$ & $\begin{array}{l}\text { RX-54-AJ } \\
1.811 \mathrm{~g} / \mathrm{cc}\end{array}$ & $\begin{array}{l}\text { on } \\
\text { Al off } \\
\text { Al off } \\
\text { Measured } \\
\end{array}$ & $\begin{array}{l}\text { on } \\
\text { on } \\
\text { Al off }\end{array}$ & $\begin{array}{l}6.62 \\
7.27 \\
7.64 \\
7.65 \\
\end{array}$ & $\begin{array}{l}\text { HMX 47.4 } \\
\text { Al 28.4 } \\
\text { TMETN } 16.1 \\
\text { NC 8 } \\
\end{array}$ & offoff \\
\hline & $\begin{array}{l}\mathrm{RDX} / \mathrm{Al} \\
1.92 \mathrm{~g} / \mathrm{cc}\end{array}$ & $\begin{array}{l}\text { on } \\
\text { Al off }\end{array}$ & $\begin{array}{l}\text { on } \\
\text { on }\end{array}$ & $\begin{array}{l}6.38 \\
7.33\end{array}$ & $\begin{array}{l}\text { RDX } 62 \\
\text { Al } 35.5\end{array}$ & off \\
\hline
\end{tabular}




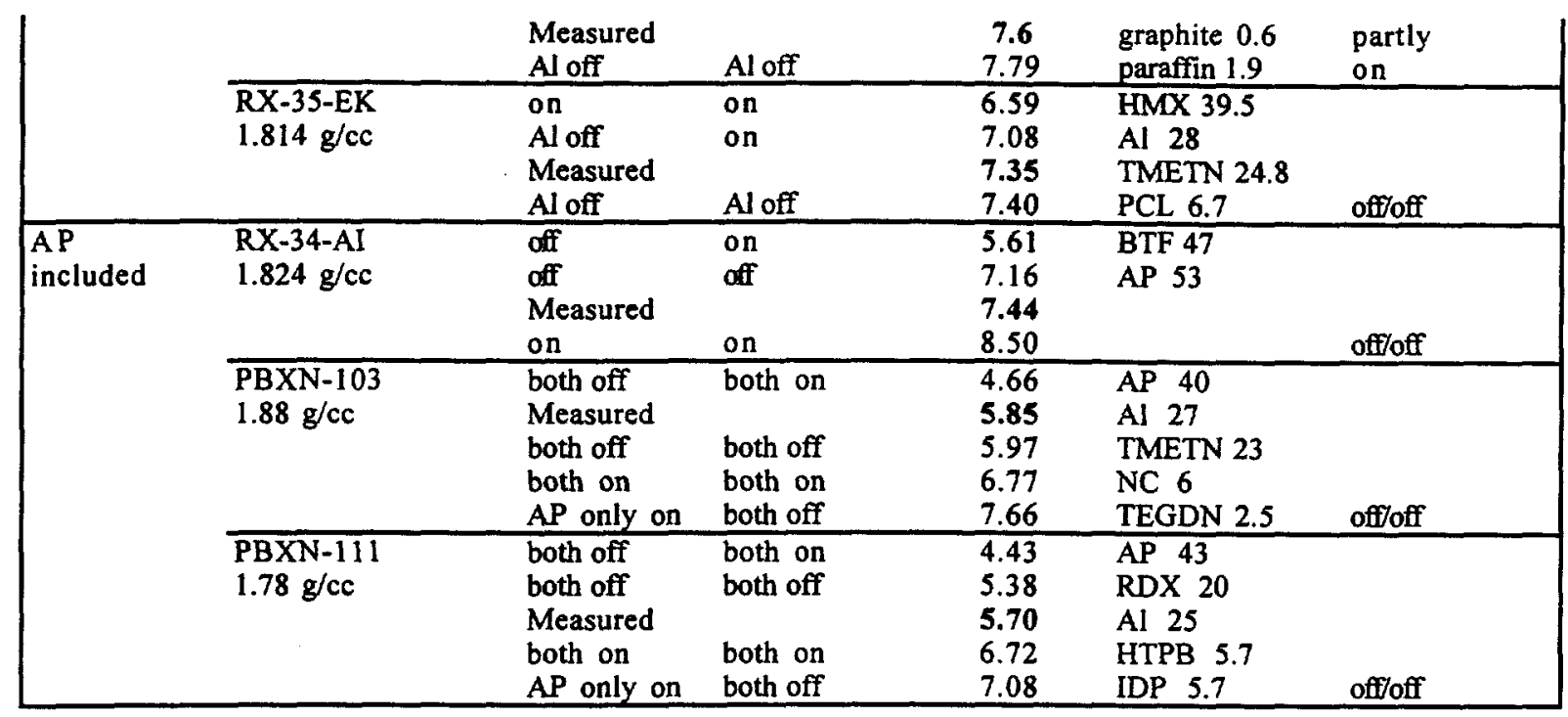

\section{REFERENCES}

1. Fried, L. E. and Souers, P. C., CHEETAH: A Next Generation Thermochemical Code, Lawrence Livermore National Laboratory report UCRL-ID-117240 (1994).

2. Fried, L. E. and Souers, P. C., "BKWC: An Empirical BKW Parametrization based on Cylinder Test Data," Propellants, Explosives, Pyrotechnics 21: 215-223 (1996).

3. Kittel, C., Introduction to Solid State Physics, 2nd ed, New York, John Wiley, 1956, p. 124.

4. Guillermet, A. F., Gustafson, P. and Hillert, M., J. Phys. Chem. Solids 46: 1427-1429 (1985).

5. Simpson, R. L., Helm, F. H., Crawford, P. C. and Kury, J. W., "Particle Size Effects in the Initiation of Explosives containing Reactive and Non-Reactive Continuous Phases," Proceedings Ninth Symposium (International) on Detonation, Portland, OR, August 28-September 1, 1989, vol. I, pp. 25-35.

6. Lawrence, W., Naval Surface Warfare Center, White Oak, MD, private communication from Jerry Forbes, 1996.

7. Forbes, J. W., Lawrence, W. and Sutherland, G. T., Naval Surface Warfare Center, White Oak, MD, private communication, 1996.

8. CPIA/M3 Solid Propellant Ingredients Manual, Chemical Propulsion Information Agency, John Hopkins University, Columbia, MD, unit 7, pp. 1 and 3.

9. Bernecker, R. and Forbes, J. W., Naval Surface Warfare Center, White Oak, MD, private communication, 1996.
10. F. Bonthoux, F., Deneuville, P. and de Longueville, Y., "Diverging Detonations in RDX and PETN Based Cast-cured PBX," Proceedings Seventh Symposium (International) on Detonation, Annapolis, MD, June 16-19, 1981, pp. 408-415.

11. Souletis, J. and Mala, J., "Influence of Test Conditions on the Ballistic Classification of Explosives," Proceedings Eighth Symposium (International) on Detonation, Albuquerque, NM, July 15-19, 1985, pp. 625-630.

12. Finger, M., Hornig, H. C., Lee, E. L. and Kury, J. W., "Metal Acceleration by Composite Explosives," Proceedings Fifth Symposium (International) on Detonation, Pasadena, CA, August 18-21, 1970, pp. 137-149.

13. Volk, F. and Schedibauer, F., "Detonation Products of less sensitive High Explosives formed under different Pressures of Argon and in Vacuum," Proceedings Ninth Symposium (International) on Detonation, Portland, OR, August 28September 1, 1989, vol. II, pp. 962-969.

14. Sutherland, G. T., Lamar, E. R., Forbes, J. W., Anderson, E., Miller, P., Ashwell, K. D., Baker, R. N. and Liddiard, T. P., "Shock Wave and Detonation Wave Response of Selected $H M X$ Based Research Explosives with HTPB Binder Systems, "High Pressure Science and Technology-1993, Proceedings American Physical Society Topical Group on Shock Compression, Colorado Springs, CO., June 28-July 2, 1993, part 2, pp. 1413-1416.

15. LLNL Cylinder Test results.

16. Forbes, J. W., Lemar, E. R., Sutherland, G. T. and Baker, R. N., Detonation Wave Curvature, Corner Turning, and Unreacted Hugoniot of $P B X N-111$, Naval Surface Warfare Center report NSWCDD/TR-92/164 (1992). 


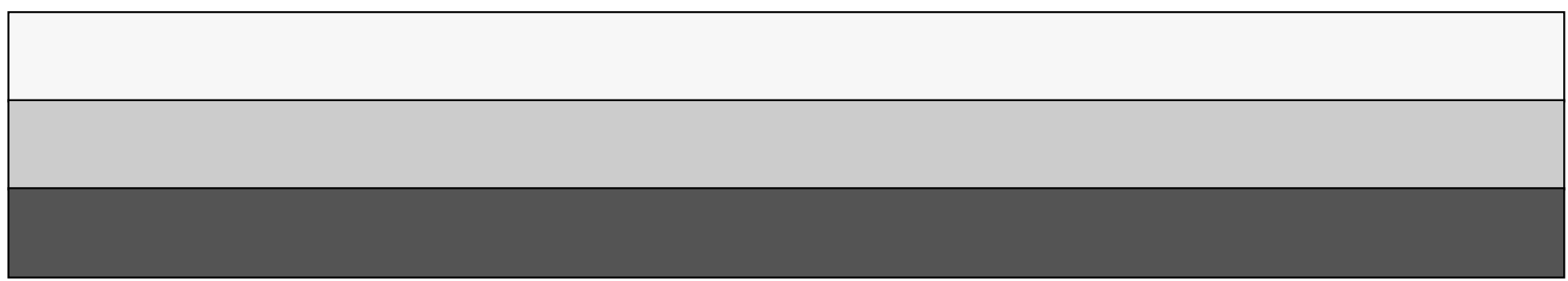

